\title{
Australian Journal of

\section{Tritipyrum (Triticum durum $\times$ Thinopyrum bessarabicum) might be able to provide an economic and stable solution against the soil salinity problem}

\author{
Maryam Kamyab ${ }^{1}$, Mohammad Kafi ${ }^{1 *}$, Hossein Shahsavand Hassani ${ }^{2}$, Morteza Goldani ${ }^{1}$, Farhad \\ Shokouhifar ${ }^{3}$
}

\author{
${ }^{1}$ Department of Agronomy and Crop Breeding, Ferdowsi University of Mashhad, Iran \\ ${ }^{2}$ Department of Agronomy and Plant Breeding, College of Agriculture, Shiraz University, Iran \\ ${ }^{3}$ Research Institute for Plant Sciences, Ferdowsi University of Mashhad, Iran
}

\section{*Corresponding author: m.kafi@um.ac.ir}

\begin{abstract}
The novel salt tolerant cereal, Tritipyrum, was suggested as a potential species for cultivation in saline soils to improve salt tolerance in wheat via breeding programs. Hence, investigation of its reaction to salinity stress, especially at different growth stages, seems to be necessary. For this purpose, an experiment was conducted using hydroponic technique in controlled as well as field conditions. Nine tritipyrum lines were tested under three levels of salinity $(50,100$ and $200 \mathrm{mM} \mathrm{NaCl})$ in hydroponic culture. Then, based on the results, three tritipyrum lines along with one wheat cultivar were cultivated in the field under three levels of salinity of irrigation water (control, $7 \mathrm{dS} / \mathrm{m}$ and $14 \mathrm{dS} / \mathrm{m}$ ). In the hydroponic test, according to the statistical analysis of the genotypes based on their vegetative, reproductive and seed maturity traits, tritipyrum lines were more salt tolerant than salt tolerant wheat cultivar. Also cluster analysis showed that $(\mathrm{St} / \mathrm{b}) \times(\mathrm{Cr} / \mathrm{b}), \mathrm{F} 4$ and $\mathrm{Az} / \mathrm{b}$ were the most salt tolerant tritipyrum lines. Furthermore, based on the field trial, tritipyrum lines showed better performance and produced higher grain yield as well as higher grain protein content than wheat cultivar in saline condition. Based on our results, it is conceivable to improve the salt tolerance of wheat through breeding programs. Tritipyrum lines can also be cultivated in saline soils as fodder or forage crop.
\end{abstract}

Keyword: Agronomic performance, salinity, tritipyrum, wheat, yield.

\section{Introduction}

The negative effect of salinity on more than 900 million hectares of lands all over the world (FAO, 2007) and the need to produce food for an expanding population (Qadir and Oster, 2004) has turned salinity into a serious problem. Many salinity management strategies such as irrigation techniques, drainage and reclamation of saline lands are costly and temporary (Singh and Singh, 2000). Genetic improvement through the selection and development of salt-resistant plants via breeding programs has been regarded as an economic, effective and stable solution for overcoming salinity and is expected to have remarkable progress in the future (Ashraf et al., 2008). Gene transfer from wild relatives to the cultivated species either through conventional crossing techniques or, if possible, genetic engineering, is highly controversial and only few salttolerant varieties have been released through this approach (Colmer et al., 2006). In this regard, tritipyrum as the third man-made amphiploid and a salt-resistant cereal is one of the plant breeding achievements to dealing with salinity stress. It is a hybridized plant from wheat and Thinopyrum Bessarabicum that can survive in half-salinity of seawater. Tritipyrum's performance and grain yield has been shown to be better than its parents and common wheat in salinity treatments (King et al., 1997). However, its potential for cultivation in saline soil has received some skepticism.

Producing genotypes with maximum economic yield under salinity stress has been considered as the main objective and an essential part of breeding programs. However, salinity tolerance is a complex trait and numerous genes are involved in salinity stress, creating different morphological, physiological and agronomical characteristics (Munns and Tester, 2008). Furthermore, the sensitivity of plants to salinity stress varies from one growth stage to another. In other words, resistance to salinity at one step does not guarantee salt-tolerance at the next phase (Lauchli and Grattan, 2007). Therefore, recognizing the most salt-tolerant genotypes at each stage, understanding the effects of salinity on different developmental growth stages of plants, and identifying a collection of credible criteria related to salinity resistance are necessary for developing management strategies and facilitating breeding programs in order to reduce the harmful effects of salinity, retain plant performance at a satisfactory level, and improve salt tolerance of plants (Zeng et al., 2002; Zeng and Shannon, 2000).

Few studies have focused on the salinity tolerance of tritipyrum lines in hydroculture experiments (Kamyab et al., 
2016). However, field trail is essential to confirm the potential of these hybrids for agricultural use and to answer the query of whether tritipyrum lines can produce commodious yield in the field condition during salinity stress. Thus, the major changes in the agronomical traits of tritipyrum lines under field condition need to be evaluated. Few research has to date investigated the salinity tolerance of tritipyrum lines in field condition (kamyab et al., 2016). Accordingly, the current study is an attempt to achieve the following objectives:

- Identifying the morphological and agronomical traits of tritipyrum lines to salinity stress in comparison with wheat

- Assessing the salinity tolerance of tritipyrum lines at vegetative, reproductive and grain maturity stages using multivariate analysis

- Investigating the effect of salinity on the agronomic traits and grain yield of tritipyrum lines in field condition.

\section{Results}

\section{Salinity effects in greenhouse experiment}

The vegetative parameters including flag leaf length, flag leaf area, number of leaf per main stem, stem length and number of tillers per plant reduced significantly due to salinity stress (Table 1). At all salinity levels and among all tritipyrum lines, Az/b showed the highest flag leaf area and length, whereas the lowest of these traits belonged to $(\mathrm{Ka} / \mathrm{b}) \times(\mathrm{Cr} / \mathrm{b}), \mathrm{F} 6$ (Table 1$)$. In tritipyrum lines, the salt tolerance indices of flag leaf length ranged from 0.64 to 1.54 at $100 \mathrm{mM} \mathrm{NaCl}$, and 0.61 to 0.94 at $200 \mathrm{mM} \mathrm{NaCl}$ treatments (Table 2). As for flag leaf area, this index ranged from 0.60 to 1.76 at the first salinity level $(100 \mathrm{mM} \mathrm{NaCl})$ and from 0.41 to 0.95 at the second salinity level $(200 \mathrm{mM}$ $\mathrm{NaCl}$ ) (Table 2). The highest leaf number per main stem was observed in $(\mathrm{Ma} / \mathrm{b}) \times(\mathrm{Cr} / \mathrm{b}), \mathrm{F} 4(6.83)$ at $50 \mathrm{mM} \mathrm{NaCl}$ treatment, while at 100 and $200 \mathrm{mM} \mathrm{NaCl}, \mathrm{La} / \mathrm{b} \mathrm{(6.33)} \mathrm{and}$ $\mathrm{Cr} / \mathrm{b}$ (6.07) lines had the highest leaf number per main stem, respectively (Table 1 ). Salt tolerance indices for the number of leaf per main stem of tritipyrum lines ranged from 0.78 to 0.98 and 0.70 to 0.94 at $100 \mathrm{mM}$ and $200 \mathrm{mM} \mathrm{NaCl}$, respectively (Table 2$)$. At all salinity treatments, $(\mathrm{St} / \mathrm{b}) \times(\mathrm{Cr} / \mathrm{b})$, F4 was the longest genotype, while La (4B/4D)/b was significantly shorter than the other genotypes (Table 1). At the first salinity treatment $(100 \mathrm{mM})$, salt tolerance indices ranged from 0.45 to 1.34 for the stem length of tritipyrum lines, while they ranged from 0.42 to 1.31 at the second salinity level (Table 2). The highest and lowest number of tiller per plant belonged to $(\mathrm{Ka} / \mathrm{b}) \times(\mathrm{Cr} / \mathrm{b}), \mathrm{F} 2$ and $\mathrm{La}$ $(4 B / 4 D) / b$, respectively (Table 1$)$. In the case of the number of tillers per plant for tritipyrum lines, salt tolerance indices were from 0.56 to 1.16 in the first salinity treatment and from 0.42 to 1.02 in the second salinity treatment (Table 2). Based on the studied vegetative traits, cluster analyses were carried out using Ward's algorithm to group the genotypes according to the similarity of their vegetative parameters at $100 \mathrm{mM}$ and $200 \mathrm{mM}$ salinity treatment (Table3). Under 100 $\mathrm{mM}$ salinity treatment, four major clusters comprising 1, 3, 3 and 2 members were determined. In $200 \mathrm{mM}$ salinity treatment, 9 genotypes were grouped into three clusters with 3, 4 and 2 members (Table 3 ). According to the results of cluster analysis for vegetative traits, $(\mathrm{St} / \mathrm{b}) \times(\mathrm{Cr} / \mathrm{b}), \mathrm{F} 4, \mathrm{Az} / \mathrm{b}$, $(\mathrm{Ma} / \mathrm{b}) \times(\mathrm{Cr} / \mathrm{b}), \mathrm{F} 4$ and $\mathrm{La} / \mathrm{b}$ were ranked as salt-tolerant lines, while $\mathrm{Cr} / \mathrm{b}$ and $\mathrm{La}(4 \mathrm{~B} / 4 \mathrm{D}) / \mathrm{b}$ were found to be salt-sensitive (Table 3). As for the salt-tolerant tritipyrum lines, flag leaf length, flag leaf area, number of leaf per main stem, stem length and tiller number per plant decreased by an average of $6,10,8,6$ and 17 percent, whereas these traits by 18,28 , 11,8 and 28 percent for salt-sensitive tritipyrum lines at 100 $\mathrm{mM} \mathrm{NaCl}$ compared to the control condition. At $200 \mathrm{mM}$ $\mathrm{NaCl}$, the flag leaf length, flag leaf area, leaf number per main stem, stem length and tiller number per plant of salttolerant tritipyrum lines reduced by an average of $22,29,16$, 11 and 33 percent compared to control, and these amounts were $36,45,13,15$ and 28 percent for salt-sensitive lines.

Considering the reproductive stage, the number of spikelet per spike and spike length in all genotypes decreased significantly with an increase in salt concentration (Table 1). However, the number of grain per plant and single grain weight gradually increased with an increase in salt levels up to $100 \mathrm{mM} \mathrm{NaCl}$. Subsequently, however, they decreased with an increase in salinity of up to $200 \mathrm{mM} \mathrm{NaCl}$ (Table 1). Spikes of tritipyrum lines were longer than Bam wheat cultivar. Among tritipyrum lines at all salinity levels, $\mathrm{Cr} / \mathrm{b}$ had the shortest spike. The longest spike at $50 \mathrm{mM}$ belonged to $\mathrm{Ka} / \mathrm{b}$, while $(\mathrm{St} / \mathrm{b}) \times(\mathrm{Cr} / \mathrm{b})$, F4had the longest spike at 100 and $200 \mathrm{mM} \mathrm{NaCl}$ (Table 1). Salt tolerance indices of the spike length of tritipyrum lines were from 0.79 to 1.04 and 0.73 to 0.96 at 100 and $200 \mathrm{mM} \mathrm{NaCl}$ treatments, respectively (Table 2). (St/b) $\times(\mathrm{Cr} / \mathrm{b}), \mathrm{F} 4$ produced the highest number of spikelets per spike, while $\mathrm{La}(4 \mathrm{~B} / 4 \mathrm{D}) / \mathrm{b}$ had the lowest one at all salinity levels (Table 1 ). During the first salinity treatment $(100 \mathrm{mMNaCl})$, salt tolerance indices in tritipyrum lines ranged from 0.72 to 1.10 for the number of spikelets per spike and from 0.70 to 0.99 at the second salinity level (200 $\mathrm{mM} \mathrm{NaCl}$ ) (Table 2). At 50 and $100 \mathrm{mM} \mathrm{NaCl}, \mathrm{Ka} / \mathrm{b}$ had the maximum number of grains per plant, while the maximum amount at $200 \mathrm{mM} \mathrm{NaCl}$ was observed in $(\mathrm{St} / \mathrm{b}) \times(\mathrm{Cr} / \mathrm{b}), \mathrm{F} 4$. La $(4 B / 4 D) / b$ had the minimum number of grains per plant at all salinity levels (Table 1 ). The amount of salt tolerance indices for the number of grains per plant fell between 0.64 and 1.47 at $100 \mathrm{mM} \mathrm{NaCl}$ and between 0.30 and 1.12 at 200 $\mathrm{mM} \mathrm{NaCl}$ in tritipyrum lines (Table 2). Additionally, the heaviest grains were observed in $\mathrm{La} / \mathrm{b}$ and $(\mathrm{St} / \mathrm{b}) \times(\mathrm{Cr} / \mathrm{b}), \mathrm{F} 4$. The lightest grains, however, belonged to $L a(4 B / 4 D) / b$ at all salinity regimes (Table 1 ). In the case of single seed weight, salt tolerance indices were from 0.68to 1.24 in the first salinity treatment. As for the second salinity treatment, salt tolerance indices ranged from 0.51 to 0.99 (Table 2). In order to group the lines according to their reproductive traits, the studied reproductive parameters were used as variables for cluster analysis (Table 4). Four clusters (including 1, 4, 3 and 1 members) were found at $100 \mathrm{mM}$ salinity treatment and four clusters (including 3, 3, 2 and 1 members) were obtained at $200 \mathrm{mM}$ of salt stress (Table 4). According to the results of cluster analysis, $(\mathrm{St} / \mathrm{b}) \times(\mathrm{Cr} / \mathrm{b}), \mathrm{F} 4,(\mathrm{Ma} / \mathrm{b}) \times(\mathrm{Cr} / \mathrm{b})$, $\mathrm{F} 4, \mathrm{La} / \mathrm{b}$ and $\mathrm{Az} / \mathrm{b}$ are most tolerant lines to salinity stress, whereas $\mathrm{Cr} / \mathrm{b}$ and $\mathrm{La}(4 \mathrm{~B} / 4 \mathrm{D}) / \mathrm{b}$ were the most sensitive lines (Table 4). On average, the number of spikelet per spike and spike length decreased by about $8 \%$ and $5 \%$ in salt-tolerant lines, while these amounts were $4 \%$ and $9 \%$ for salt-sensitive lines at $100 \mathrm{mM} \mathrm{NaCl}$ treatment. At $200 \mathrm{mM} \mathrm{NaCl}$, the decrease in the number of spikelet per spike and spike length of tolerant lines were $17 \%$ and $10 \%$ and for sensitive lines were $11 \%$ and $14 \%$, respectively. The number of seeds per plant, single seed weight as well as seed length and 
width of salt-tolerant tritipyrum lines enhanced with an increase in salinity up to $100 \mathrm{mM} \mathrm{NaCl}$ by an average of 26 , 13,7 and 13 percent, respectively, while increasing salinity up to $100 \mathrm{mM} \mathrm{NaCl}$ caused the reduction of these traits in salt-sensitive lines. The reduction of the number of seed per plant, single seed weight and seed width of salt-sensitive tritipyrum lines at $100 \mathrm{mM} \mathrm{NaCl}$ were 6,7 and 40 percent , but seed length of these lines increased about 3\%. At $200 \mathrm{mM} \mathrm{NaCl}$, the number of seed per plant and single seed weight reduced by an average of $18 \%$ and $10 \%$ for salttolerant tritipyrum lines and reduced by about $30 \%$ and $40 \%$ for salt-sensitive lines.

Tritipyrum lines exhibited a better performance against salinity stress compared to Bam wheat cultivar and their grain yield per plant was more than wheat, especially at 100 and $200 \mathrm{mM} \rightarrow \mathrm{NaCl}$ levels (Fig. 1). At $50 \mathrm{mM}$ salinity treatment, primary tritipyrum lines produced similar or even lower grain yield per plant in comparison with Bam wheat cultivar. However, at this salinity level, La (4B, 4D)/b had the lowest $(0.65 \mathrm{~g} /$ plant $)$ and $\mathrm{Ka} / \mathrm{b}$ had the highest $(1.18 \mathrm{~g} /$ plant $)$ grain yield per plant (Fig.1). At the second salinity treatment $(100 \mathrm{mM} \mathrm{NaCl})$, grain yield per plant of tritipyrum lines was satisfactory, while there was a significant decrease in the yield of Bam wheat cultivar (Fig.1). Except for La (4B, 4D)/b and $\mathrm{Cr} / \mathrm{b}$, other tritipyrum lines produced higher grain yield than Bam wheat cultivar in $100 \mathrm{mM} \mathrm{NaCl}$. At this salinity level, the highest grain yield belonged to $(\mathrm{St} / \mathrm{b}) \times(\mathrm{Cr} / \mathrm{b}), \mathrm{F} 4$ and the lowest yield was attributed to $L a(4 B, 4 D) / b$ lines (Fig.1). At the highest salinity level $(200 \mathrm{mM} \mathrm{NaCl})$, all primary tritipyrum lines produced grain yield, but Bam wheat cultivar did not set any seeds (Fig.1). At this salinity level, $A z / b$ produced the highest grain yield, La $(4 B, 4 D) / b$ had the lowest and Bam wheat cultivar produced no grain yield at all (Fig.1). Tritipyrum lines and Bam wheat cultivar experienced a $37 \%$ and $100 \%$ reduction in grain yield per plant at $200 \mathrm{mM} \mathrm{NaCl}$ compared to $50 \mathrm{mM} \mathrm{NaCl}$, respectively (Fig. 1). Salt tolerance indices of grain yield ranged from 0.40 to 1.69 and from 0.19 to 0.97 at the first and second salinity levels, respectively (Table 2). Cluster analysis based on grain yield ranked the genotypes in four groups with 2, 5, 1 and 1 members at $100 \mathrm{mM} \mathrm{NaCl}$ and in five groups with 4, 1, 2, 1 and 1 members at $200 \mathrm{mM} \mathrm{NaCl}$. Based on grain yield, $(\mathrm{St} / \mathrm{b}) \times(\mathrm{Cr} / \mathrm{b}), \mathrm{F} 4$ and $\mathrm{Az} / \mathrm{b}$ were salttolerant, whereas $\mathrm{Cr} / \mathrm{b}$ and $\mathrm{La}(4 \mathrm{~B} / 4 \mathrm{D}) / \mathrm{b}$ were salt-sensitive (Table 5). Compared to $50 \mathrm{mM} \mathrm{NaCl}$, the grain yield of salttolerant lines increased by about $40.7 \%$ at $100 \mathrm{mM} \mathrm{NaCl}$ and decreased by $20.3 \%$ at $200 \mathrm{mM} \mathrm{NaCl}$. However, salt-sensitive tritipyrum lines demonstrated $15.4 \%$ and $55.3 \%$ decrease at 100 and $200 \mathrm{mM} \mathrm{NaCl}$, respectively.

\section{Salinity stress effects under field conditions}

In order to complement and confirm the hydrucultural results, a field experiment was conducted. Number of total and fertile tillers per plant, number of seeds per plant, spikes weight per plant, number of spikes and biological yield were significantly higher in tritipyrum lines compared to Bam wheat cultivar (Table 6). In tritipyrum lines, these traits increased following an increase in salinity level up to $7 \mathrm{dS} / \mathrm{m}$. Then, they decreased at $14 \mathrm{dS} / \mathrm{m}$. However, the increase of salinity caused a decrease in these traits for Bam wheat cultivar (Table 6). The highest degree of these traits belonged to $(\mathrm{St} / \mathrm{b}) \times(\mathrm{Cr} / \mathrm{b}), \quad \mathrm{F} 4$ at the second salinity treatment $(7 \mathrm{dS} / \mathrm{m})$, while the lowest degree was observed in Bam wheat cultivar at $14 \mathrm{dS} / \mathrm{m}$ of salinity (Table 6). For all the genotypes, spike length and number of spikelet per spike decreased with an increase in salt concentration (Table 6). $(\mathrm{St} / \mathrm{b}) \times(\mathrm{Cr} / \mathrm{b}), \mathrm{F} 4$ and Bam wheat cultivar had the highest and lowest value of spike length, respectively. Furthermore, Bam wheat cultivar produced the highest number of spikelet per spike in the control condition, whereas $(\mathrm{Ka} / \mathrm{b}) \times(\mathrm{Cr} / \mathrm{b}), \mathrm{F} 4$ exhibited the lowest number of spikelet per spike as compared to the other genotypes (Table 6). Increasing salinity up to $7 \mathrm{dS} / \mathrm{m}$ led to an increase in the harvest index and biological yield of tritipyrum lines, while these traits decreased by increasing salinity up to $14 \mathrm{dS} / \mathrm{m}$; however, the increase of salinity reduced this trait in Bam wheat cultivar (Table 6). The harvest index of Bam wheat cultivar was higher than tritipyrum lines (Table 6).

Increasing salinity level caused a reduction in the stem height and peduncle length of all genotypes (Fig.2 A $\neg-B$ ). $(\mathrm{St} / \mathrm{b}) \times(\mathrm{Cr} / \mathrm{b}), \mathrm{F} 4$ line was the highest in stem height and peduncle length, while $(\mathrm{Ka} / \mathrm{b}) \times(\mathrm{Cr} / \mathrm{b}), \mathrm{F} 6$ and Bam wheat cultivar were the shortest in these two traits (Fig. $2 \mathrm{~A} \neg-\mathrm{B}$ ).

Tritipyrum lines had significantly greater straw weight than Bam wheat cultivar (Fig. 3 A). In tritipyrum lines, increasing salinity up to $7 \mathrm{dS} / \mathrm{m}$ led to an increase in straw weight, while $14 \mathrm{dS} / \mathrm{m}$ salinity resulted in a decrease in this trait. However, in Bam wheat cultivar, increasing the level of salinity caused a reduction in straw weight (Fig. $3 \mathrm{~A}$ ). The highest straw weight was exhibited by $(\mathrm{St} / \mathrm{b}) \times(\mathrm{Cr} / \mathrm{b}), \mathrm{F} 4$ at $7 \mathrm{dS} / \mathrm{m}$ and the lowest amount was attributed to Bam wheat cultivar under $14 \mathrm{dS} / \mathrm{m}$ salinity (Fig. $3 \mathrm{~A}$ ).

In field condition, the performance of tritipyrum lines was less affected by salinity in comparison to wheat (Fig. 3 B). Overall, yield and its component in addition to the agronomic traits of tritipyrum lines were superior to Bam wheat cultivar under saline conditions, especially at $7 \mathrm{dS} / \mathrm{m}$ $\mathrm{NaCl}$ (Supplementary Fig. 1). Under salinity condition, the grain yield of tritipyrum lines, especially $(\mathrm{St} / \mathrm{b}) \times(\mathrm{Cr} / \mathrm{b})$, F4 and $\mathrm{Az} / \mathrm{b}$, were higher than Bam wheat cultivar (Fig.3 B). Increasing the level of salinity led to a reduction in the grain yield of Bam wheat cultivar, while it increased the grain yield of tritipyrum lines at $7 \mathrm{dS} / \mathrm{m}$ and then decreased grain yield at $14 \mathrm{dS} / \mathrm{m}$ (Fig.3 B).

Increasing salinity reduced the grain protein content of all the genotypes (Fig.3 C). Grain protein content of tritipyrum lines was higher than that of Bam wheat cultivar. Moreover, $(\mathrm{St} / \mathrm{b}) \times(\mathrm{Cr} / \mathrm{b}), \quad \mathrm{F} 4$ showed the highest amount of grain protein content at all salinity levels (Fig.3 C).

\section{Discussion}

Tritipyrum is a result of breeders' efforts to genetically improve the salt resistance of wheat. Also, due to its high salinity tolerance and possibility of using it for increasing the genetic salinity resistance of common wheat (King et al., 1997), it seems necessary to investigate its response to salinity stress.

Salinity stress affects the phenotypic characteristics, which may be exhibited through growth reduction and/or death of wheat genotypes (Parida and Das, 2005). The upper leaves of wheat main stem, especially flag leaf, were suggested as source potential location for supplying photo assimilate so 
Table 1. Effect of salinity on agronomic parameters and spike characteristics of tritipyrum lines and wheat cultivar Bam in green house experiment.

\begin{tabular}{|c|c|c|c|c|c|c|c|c|c|c|}
\hline Genotypes & SL & FLL & FLA & LN & $\mathrm{MSH}$ & TN & $\mathrm{SL}$ & SNS & SNP & SSW \\
\hline \multirow[t]{3}{*}{$(\mathrm{Ka} / \mathrm{b})^{*}(\mathrm{Cr} / \mathrm{b}), \mathrm{F}_{2}$} & 50 & $12.58^{\mathrm{dk}}$ & $7.15^{\mathrm{cg}}$ & $6.50^{\mathrm{ac}}$ & $58.50^{\mathrm{ej}}$ & $5.33^{\mathrm{a}}$ & $10.67^{\mathrm{ae}}$ & $9.67^{\mathrm{im}}$ & $25.03^{\mathrm{bt}}$ & $36.24^{\text {eh }}$ \\
\hline & 100 & $12.15^{\mathrm{tk}}$ & $6.67^{\mathrm{dg}}$ & $5.50^{\mathrm{ae}}$ & $57.20^{\mathrm{fk}}$ & $4.17^{\mathrm{ab}}$ & $9.17^{\mathrm{cf}}$ & $9.23^{\mathrm{jo}}$ & $29.67^{b}$ & $42.95^{\text {ad }}$ \\
\hline & 200 & $11.33^{i k}$ & $5.73^{\mathrm{fg}}$ & $4.67^{e}$ & $54.67^{\mathrm{gk}}$ & $3.67^{\mathrm{ad}}$ & $8.67^{d t}$ & $9.07^{\mathrm{ko}}$ & $20.43^{\mathrm{fi}}$ & $34.84^{\text {th }}$ \\
\hline \multirow[t]{3}{*}{$(\mathrm{Ka} / \mathrm{b})^{*}(\mathrm{Cr} / \mathrm{b}), \mathrm{F}_{6}$} & 50 & $12.08^{\mathrm{gk}}$ & $6.44^{\mathrm{eg}}$ & $6.50^{\mathrm{ac}}$ & $54.33^{\mathrm{gk}}$ & $3.50^{\text {ad }}$ & $11.67^{\mathrm{ac}}$ & $10.47^{\dagger j}$ & $23.73^{\mathrm{cg}}$ & $36.20^{\mathrm{eh}}$ \\
\hline & 100 & $11.57^{\mathrm{ik}}$ & $6.09^{f g}$ & $5.50^{\mathrm{ae}}$ & $52.00^{\mathrm{hk}}$ & $3.17^{\text {be }}$ & $10.93^{\mathrm{ae}}$ & $10.00^{\mathrm{hl}}$ & $25.80^{\text {be }}$ & $42.46^{\text {ad }}$ \\
\hline & 200 & $9.53^{k}$ & $4.12^{\mathrm{g}}$ & $4.50^{e}$ & $45.17^{\mathrm{kl}}$ & $2.77^{\text {be }}$ & $10.17^{\mathrm{ae}}$ & $9.73^{\mathrm{im}}$ & $20.83^{\mathrm{fi}}$ & $35.43^{\text {th }}$ \\
\hline \multirow[t]{3}{*}{$(\mathrm{Ma} / \mathrm{b})^{*}(\mathrm{Cr} / \mathrm{b}), \mathrm{F}_{4}$} & 50 & $16.52^{c}$ & $9.98^{\mathrm{cd}}$ & $6.83^{\mathrm{a}}$ & $63.33^{\mathrm{dh}}$ & $3.50^{\mathrm{ad}}$ & $10.92^{\mathrm{ae}}$ & $11.53^{\mathrm{cf}}$ & $20.53^{\mathrm{fi}}$ & $36.83^{\mathrm{dh}}$ \\
\hline & 100 & $15.23^{\mathrm{cf}}$ & $8.72^{\mathrm{cf}}$ & $5.93^{\mathrm{ae}}$ & $61.67^{\mathrm{di}}$ & $2.60^{\text {be }}$ & $10.50^{\mathrm{ae}}$ & $11.10^{\mathrm{eh}}$ & $28.77^{b}$ & $41.53^{b f}$ \\
\hline & 200 & $12.17^{\mathrm{tk}}$ & $6.87^{\mathrm{cg}}$ & $5.67^{\mathrm{ae}}$ & $59.42^{\mathrm{ej}}$ & $2.20^{\mathrm{ce}}$ & $9.87^{\text {be }}$ & $11.23^{\mathrm{dh}}$ & $18.33^{\mathrm{hj}}$ & $28.91^{\mathrm{jk}}$ \\
\hline \multirow[t]{3}{*}{$(\mathrm{St} / \mathrm{b})^{*}(\mathrm{Cr} / \mathrm{b}), \mathrm{F}_{4}$} & 50 & $14.20^{\mathrm{ci}}$ & $8.77^{\mathrm{ct}}$ & $6.33^{\mathrm{ad}}$ & $95.00^{\mathrm{a}}$ & $2.83^{\text {be }}$ & $12.33^{\mathrm{ab}}$ & $13.53^{\mathrm{a}}$ & $25.93^{\text {be }}$ & $37.62^{\mathrm{ch}}$ \\
\hline & 100 & $13.43^{\mathrm{cj}}$ & $8.08^{c f}$ & $5.73^{\mathrm{ae}}$ & $82.58^{\mathrm{ab}}$ & $2.53^{\text {be }}$ & $11.67^{\mathrm{ac}}$ & $12.60^{\mathrm{ac}}$ & $34.67^{a}$ & $48.12^{\mathrm{a}}$ \\
\hline & 200 & $12.07^{\mathrm{gk}}$ & $6.81^{\mathrm{dg}}$ & $5.50^{\mathrm{ae}}$ & $80.67^{b c}$ & $2.17^{\mathrm{ce}}$ & $10.58^{\mathrm{ae}}$ & $11.33^{\mathrm{cg}}$ & $21.67^{\mathrm{ei}}$ & $35.03^{g i}$ \\
\hline \multirow[t]{3}{*}{$\mathrm{Az} / \mathrm{b}$} & 50 & $25.82^{\mathrm{b}}$ & $21.35^{\mathrm{a}}$ & $5.50^{\mathrm{ae}}$ & $61.50^{\mathrm{di}}$ & $3.67^{\mathrm{ad}}$ & $10.58^{\mathrm{ae}}$ & $12.47^{\text {ad }}$ & $27.53^{\mathrm{bd}}$ & $39.61^{\mathrm{bh}}$ \\
\hline & 100 & $24.18^{\mathrm{bk}}$ & $17.83^{b}$ & $5.30^{\mathrm{ae}}$ & $59.83^{\mathrm{ej}}$ & $3.27^{\text {be }}$ & $10.07^{\mathrm{ae}}$ & $10.67^{f i}$ & $29.40^{\mathrm{bc}}$ & $40.89^{\mathrm{bg}}$ \\
\hline & 200 & $14.70^{\mathrm{ch}}$ & $9.59^{\mathrm{ce}}$ & $4.50^{\mathrm{e}}$ & $56.08^{\mathrm{fk}}$ & $3.17^{\text {be }}$ & $9.50^{c f}$ & $9.47^{\text {in }}$ & $21.23^{\mathrm{ei}}$ & $37.61^{\mathrm{ch}}$ \\
\hline \multirow[t]{3}{*}{$\mathrm{Cr} / \mathrm{b}$} & 50 & $14.02^{\mathrm{cj}}$ & $8.96^{\mathrm{cf}}$ & $6.67^{\mathrm{ab}}$ & $63.92^{\mathrm{dh}}$ & $3.83^{\mathrm{ac}}$ & $10.08^{\mathrm{ae}}$ & $11.60^{\mathrm{cf}}$ & $12.17^{\mathrm{ei}}$ & $35.24^{\text {gh }}$ \\
\hline & 100 & $10.08^{k}$ & $6.12^{\mathrm{fg}}$ & $5.97^{\mathrm{ae}}$ & $58.50^{\mathrm{ej}}$ & $2.67^{\text {be }}$ & $8.83^{\mathrm{df}}$ & $11.10^{\mathrm{eh}}$ & $22.83^{\mathrm{ch}}$ & $38.31^{\mathrm{ch}}$ \\
\hline & 200 & $9.95^{k}$ & $5.57^{\mathrm{tg}}$ & $6.07^{\text {ae }}$ & $49.83^{\mathrm{hk}}$ & $2.23^{\mathrm{ce}}$ & $8.17^{\mathrm{eg}}$ & $9.53^{\text {in }}$ & $19.57^{\mathrm{gj}}$ & $23.52^{\mathrm{kl}}$ \\
\hline \multirow[t]{3}{*}{$\mathrm{Ka} / \mathrm{b}$} & 50 & $14.78^{\mathrm{cj}}$ & $9.52^{\mathrm{ce}}$ & $6.17^{\text {ae }}$ & $51.83^{\text {hk }}$ & $3.50^{\mathrm{ad}}$ & $12.67^{\mathrm{a}}$ & $12.50^{\text {ad }}$ & $29.47^{b}$ & $39.37^{\mathrm{bh}}$ \\
\hline & 100 & $13.33^{\mathrm{cj}}$ & $6.32^{\mathrm{eg}}$ & $5.00^{\text {be }}$ & $47.92^{\mathrm{jk}}$ & $3.17^{\text {be }}$ & $9.67^{\text {be }}$ & $11.53^{\mathrm{cf}}$ & $35.03^{\mathrm{a}}$ & $43.01^{\mathrm{ac}}$ \\
\hline & 200 & $10.95^{j k}$ & $5.95^{\mathrm{fg}}$ & $4.50^{\mathrm{e}}$ & $44.67^{\mathrm{kl}}$ & $2.67^{\text {be }}$ & $8.17^{\mathrm{eg}}$ & $10.20^{\mathrm{gk}}$ & $21.33^{\mathrm{ei}}$ & $28.92^{i k}$ \\
\hline \multirow[t]{3}{*}{$\mathrm{La} / \mathrm{b}$} & 50 & $15.47^{\mathrm{cd}}$ & $10.21^{c}$ & $6.83^{\mathrm{a}}$ & $74.50^{\text {bd }}$ & $3.50^{\mathrm{ad}}$ & $11.33^{\mathrm{ad}}$ & $13.17^{\mathrm{ab}}$ & $21.67^{\mathrm{ei}}$ & $42.24^{\mathrm{ae}}$ \\
\hline & 100 & $14.93^{\mathrm{cf}}$ & $9.67^{\mathrm{ce}}$ & $6.33^{\mathrm{ad}}$ & $70.83^{\text {be }}$ & $2.74^{\text {be }}$ & $10.50^{\mathrm{ae}}$ & $12.17^{\text {be }}$ & $26.20^{\mathrm{bd}}$ & $44.95^{\mathrm{ab}}$ \\
\hline & 200 & $12.07^{\mathrm{gk}}$ & $6.85^{\mathrm{dg}}$ & $5.50^{\mathrm{ae}}$ & $66.83^{\mathrm{dg}}$ & $2.43^{\text {be }}$ & $10.33^{\mathrm{ae}}$ & $9.73^{\mathrm{im}}$ & $16.90^{\mathrm{ij}}$ & $39.04^{\mathrm{bh}}$ \\
\hline \multirow[t]{3}{*}{$\mathrm{La}(4 \mathrm{~b}, 4 \mathrm{~d}) / \mathrm{b}$} & 50 & $15.75^{\mathrm{cd}}$ & $8.62^{\mathrm{cf}}$ & $6.67^{\mathrm{ab}}$ & $30.00^{\mathrm{m}}$ & $2.67^{\text {be }}$ & $11.00^{\text {ad }}$ & $8.53^{\mathrm{mo}}$ & $18.90^{\mathrm{gj}}$ & $34.49^{\mathrm{hj}}$ \\
\hline & 100 & $14.33^{\mathrm{ci}}$ & $6.53^{\mathrm{eg}}$ & $5.97^{\text {ae }}$ & $27.53^{\mathrm{m}}$ & $2.00^{\mathrm{ce}}$ & $10.47^{\mathrm{ae}}$ & $8.27^{\text {no }}$ & $15.23^{\mathrm{j}}$ & $26.43^{k}$ \\
\hline & 200 & $11.67^{\mathrm{hk}}$ & $6.05^{\mathrm{tg}}$ & $5.67^{\mathrm{ae}}$ & $26.00^{\mathrm{m}}$ & $1.50^{\mathrm{e}}$ & $9.87^{\text {be }}$ & $8.10^{\circ}$ & $8.97^{k}$ & $18.84^{\prime}$ \\
\hline \multirow[t]{3}{*}{ Wheat cultivar Bam } & 50 & $29.20^{\mathrm{a}}$ & $23.00^{\mathrm{a}}$ & $5.50^{\mathrm{ae}}$ & $68.92^{\mathrm{cf}}$ & $2.67^{\text {be }}$ & $6.75^{\mathrm{fh}}$ & $10.17^{\mathrm{gk}}$ & $29.50^{b}$ & $41.91^{\text {be }}$ \\
\hline & 100 & $23.88^{b}$ & $16.15^{\mathrm{b}}$ & $4.83^{\mathrm{ce}}$ & $61.00^{\mathrm{ej}}$ & $1.83^{\mathrm{de}}$ & $5.67^{\text {gh }}$ & $9.33^{\mathrm{jo}}$ & $21.67^{\mathrm{ei}}$ & $40.12^{\text {bh }}$ \\
\hline & 200 & $15.32^{\mathrm{ce}}$ & $7.75^{\mathrm{cf}}$ & $4.50^{e}$ & $38.33^{\operatorname{lm}}$ & $1.50^{\mathrm{e}}$ & $5.17^{\text {lh }}$ & $8.87^{10}$ & 0.00 & 0.00 \\
\hline
\end{tabular}
per spike, SNP: seed number per plant, SSW: single seed width $(\mathrm{cm})$. Different letters indicate statistically different means $(\mathrm{p} \leq 0.05)$.

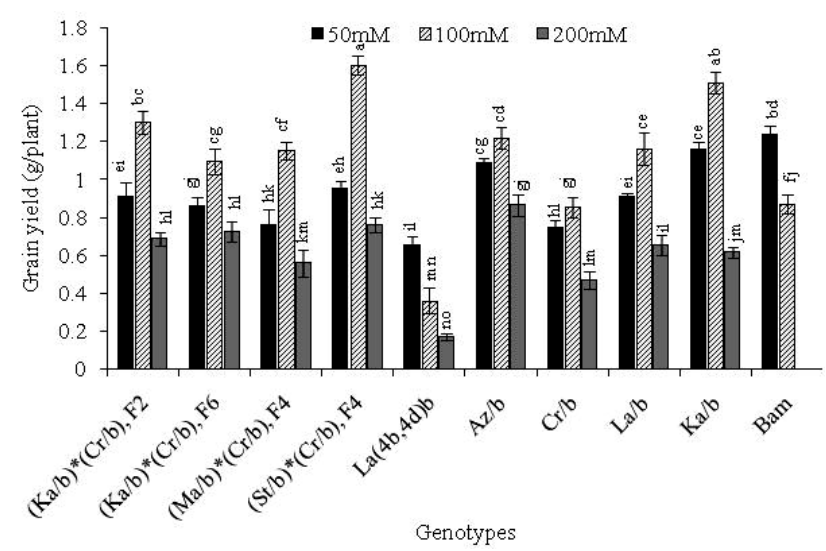

Fig 1. Effect of salinity stress on grain yield of tritipyrum lines in green house experiment. Different letters indicate statistically different means $(p \leq 0.05)$. 
Table 2. Salt tolerant indices of agronomic traits of tritipyrum lines at different growth stage in green house experiment.

\begin{tabular}{lccccccccccc}
\hline Genotypes & SL & FLL & FLA & LN & MSH & TN & SL & SNS & SNP & SSW & GY \\
\hline$(\mathrm{Ka} / \mathrm{b})^{*}(\mathrm{Cr} / \mathrm{b}), \mathrm{F}_{2}$ & 100 & 0.74 & 0.60 & 0.85 & 0.85 & 0.88 & 0.97 & 0.87 & 1.09 & 1.17 & 1.23 \\
& 200 & 0.61 & 0.41 & 0.70 & 0.74 & 0.77 & 0.90 & 0.85 & 0.88 & 0.99 & 0.81 \\
& & & & & & & & & & & \\
$(\mathrm{Ka} / \mathrm{b})^{*}(\mathrm{Cr} / \mathrm{b}), \mathrm{F}_{6}$ & 100 & 0.77 & 0.66 & 0.85 & 0.93 & 1.16 & 0.81 & 0.80 & 1.25 & 1.24 & 1.46 \\
& 200 & 0.72 & 0.57 & 0.72 & 0.89 & 1.02 & 0.77 & 0.79 & 0.86 & 0.96 & 0.77 \\
& & & & & & & & & & & \\
$(\mathrm{Ma} / \mathrm{b})^{*}(\mathrm{Cr} / \mathrm{b}), \mathrm{F}_{4}$ & 100 & 0.97 & 0.86 & 0.92 & 1.00 & 0.72 & 0.93 & 0.97 & 1.21 & 1.18 & 1.29 \\
& 200 & 0.78 & 0.68 & 0.88 & 0.97 & 0.61 & 0.88 & 0.98 & 0.77 & 0.88 & 0.63 \\
& & & & & & & & & & & \\
$(\mathrm{St} / \mathrm{b})^{*}(\mathrm{Cr} / \mathrm{b}), \mathrm{F}_{4}$ & 100 & 0.86 & 0.80 & 0.89 & 1.34 & 0.71 & 1.04 & 1.10 & 1.46 & 0.97 & 1.79 \\
& 200 & 0.77 & 0.67 & 0.85 & 1.31 & 0.60 & 0.96 & 0.99 & 0.91 & 0.86 & 0.85 \\
$\mathrm{Az} / \mathrm{b}$ & & & & & & & & & & & \\
& 100 & 1.54 & 1.76 & 0.82 & 0.97 & 0.91 & 0.89 & 0.93 & 1.24 & 1.11 & 1.36 \\
$\mathrm{Cr} / \mathrm{b}$ & 200 & 0.94 & 0.95 & 0.70 & 0.91 & 0.88 & 0.84 & 0.82 & 0.89 & 0.89 & 0.97 \\
& & & & & & & & & & & \\
$\mathrm{Ka} / \mathrm{b}$ & 100 & 0.64 & 0.61 & 0.93 & 0.95 & 0.74 & 0.79 & 0.97 & 0.96 & 0.93 & 0.96 \\
& 200 & 0.63 & 0.57 & 0.94 & 0.81 & 0.62 & 0.73 & 0.83 & 0.82 & 0.71 & 0.53 \\
& & & & & & & & & & & \\
$\mathrm{La} / \mathrm{b}$ & 100 & 0.85 & 0.62 & 0.78 & 0.78 & 0.88 & 0.86 & 1.00 & 1.47 & 1.19 & 1.69 \\
& 200 & 0.70 & 0.59 & 0.70 & 0.73 & 0.74 & 0.73 & 0.89 & 0.90 & 0.75 & 0.69 \\
$\mathrm{La}$ & & & & & & & & & & & \\
& 100 & 0.95 & 0.96 & 0.98 & 1.15 & 0.76 & 0.93 & 1.06 & 1.10 & 0.97 & 1.30 \\
& 200 & 0.77 & 0.68 & 0.85 & 1.09 & 0.68 & 0.92 & 0.85 & 0.71 & 0.85 & 0.73 \\
& & & & & & & & & & & \\
& 100 & 0.91 & 0.65 & 0.93 & 0.45 & 0.56 & 0.93 & 0.72 & 0.64 & 0.68 & 0.40 \\
& 200 & 0.74 & 0.60 & 0.88 & 0.42 & 0.42 & 0.88 & 0.70 & 0.38 & 0.51 & 0.19 \\
& & & & & & & & & & & \\
& 100 & 2.04 & 2.97 & 0.66 & 1.18 & 0.42 & 0.33 & 0.73 & 1.09 & 1.20 & 1.25 \\
& 200 & 1.54 & 1.43 & 0.62 & 0.74 & 0.35 & 0.30 & 0.30 & 0.00 & 0.00 & 0.00 \\
\hline
\end{tabular}

SL: salinity level, FLL: flag leaf length, FLA: flag leaf area, LN: leaf number per main stem, MSH: main stem height, TN: tiller number, SL: spike length, SNS: spikelet number per spike, SNP: seed number per plant, SSW: single seed weight, GY: grain yield.
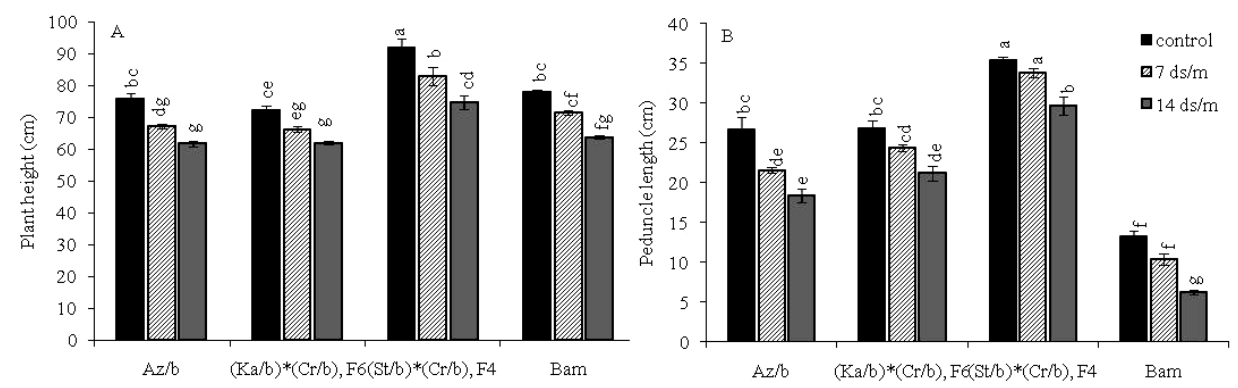

$$
\text { Genptypes }
$$

Fig 2. Salinity effect on plant height (A) and peduncle length (B) of tritipyrum lines and wheat cultivar Bam under field conditions . Different letters indicate statistically different means $(p \leq 0.05)$.

Table 3. Rankings of genotypes for their relative salt tolerance based on agronomic parameters of tritipyrum lines in green house experiment (Flag leaf length, flag leaf area, Leaf number per main stem, Main stem height and tiller number).

\begin{tabular}{|c|c|c|c|c|c|c|c|}
\hline \multirow[t]{2}{*}{ Genotypes } & & \multicolumn{2}{|c|}{$\begin{array}{r}\text { Salinity levels } \\
(\mathrm{mMNaCl})\end{array}$} & & \multirow[t]{2}{*}{ Sum } & \multirow[t]{2}{*}{$\begin{array}{r}\text { Genotype } \\
\text { ranking }\end{array}$} & \multirow[t]{2}{*}{$\begin{array}{r}\text { Tolerance } \\
\text { degree }\end{array}$} \\
\hline & & 100 & 200 & & & & \\
\hline$(\mathrm{St} / \mathrm{b})^{*}(\mathrm{Cr} / \mathrm{b}), \mathrm{F}_{4}$ & 2 & 1 & & 3 & & 1 & Tolerant \\
\hline $\mathrm{Az} / \mathrm{b}$ & 1 & 2 & & 3 & & 1 & Tolerant \\
\hline $\mathrm{Ma} / \mathrm{b}^{*} \mathrm{Cr} / \mathrm{b}, \mathrm{F}_{4}$ & 2 & 1 & & 3 & & 1 & Tolerant \\
\hline $\mathrm{La} / \mathrm{b}$ & 2 & & 1 & 3 & & 1 & Tolerant \\
\hline $\mathrm{Ka} / \mathrm{b}$ & 3 & & 2 & 5 & & 2 & Moderate \\
\hline$(\mathrm{Ka} / \mathrm{b})^{*}(\mathrm{Cr} / \mathrm{b}), \mathrm{F}_{2}$ & 3 & & 2 & 5 & & 2 & Moderate \\
\hline$(\mathrm{Ka} / \mathrm{b})^{*}(\mathrm{Cr} / \mathrm{b}), \mathrm{F}_{6}$ & 3 & & 2 & 5 & & 2 & Moderate \\
\hline $\mathrm{Cr} / \mathrm{b}$ & 4 & & 3 & 7 & & 3 & Sensitive \\
\hline $\mathrm{La}(4 \mathrm{~b}, 4 \mathrm{~d}) / \mathrm{b}$ & 4 & & 3 & 7 & & 3 & Sensitive \\
\hline
\end{tabular}




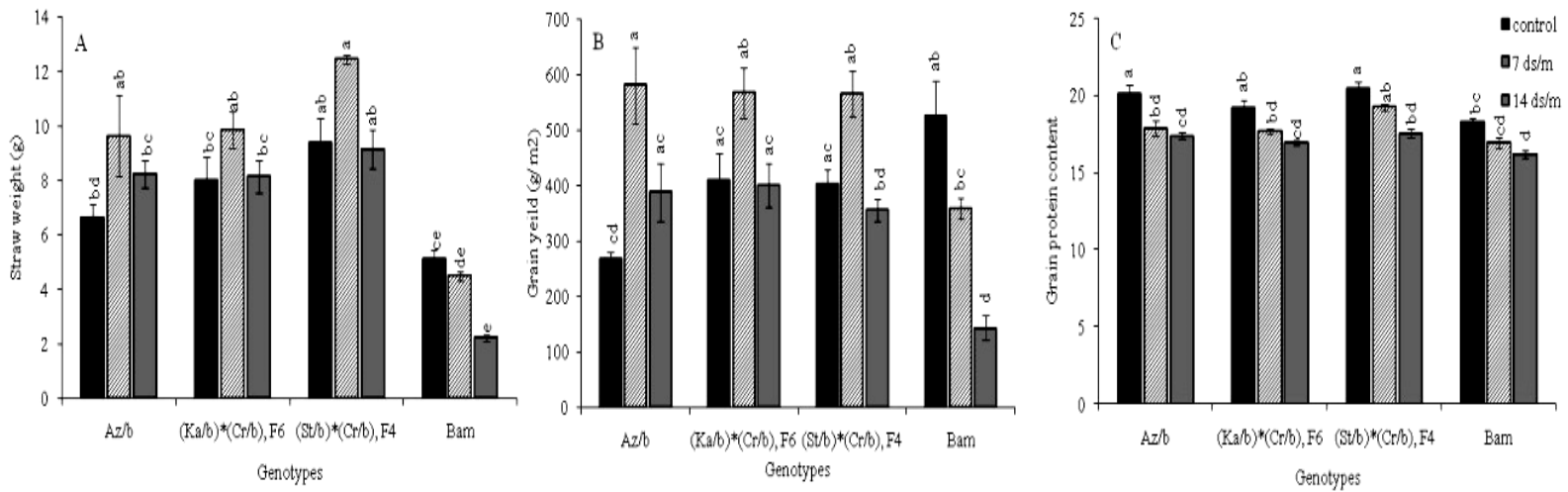

Fig 3. Effect of salinity treatment on straw weight $(A)$ grain yield $(B)$ and grain protein content $(C)$ of tritipyrum lines and wheat cultivar Bam under field condition. Different letters indicate statistically different means $(p \leq 0.05)$.

Table 4. Rankings of genotypes for their relative salt tolerance based on reproductive characteristics of tritipyrum lines in green house experiment (Number of spikelet per spike, Spike length, Seed number per plant, and Single seed weight).

\begin{tabular}{|c|c|c|c|c|c|c|c|}
\hline \multirow[t]{2}{*}{ Genotypes } & & \multicolumn{2}{|c|}{$\begin{array}{r}\text { Salinity levels } \\
(\mathrm{mMNaCl})\end{array}$} & & \multirow[t]{2}{*}{ Sum } & \multirow[t]{2}{*}{$\begin{array}{r}\text { Genotype } \\
\text { ranking }\end{array}$} & \multirow[t]{2}{*}{$\begin{array}{r}\text { Tolerance } \\
\text { degree }\end{array}$} \\
\hline & & 100 & 200 & & & & \\
\hline$(\mathrm{St} / \mathrm{b})^{*}(\mathrm{Cr} / \mathrm{b}), \mathrm{F}_{4}$ & 1 & 1 & & 2 & 1 & & Tolerant \\
\hline $\mathrm{Az} / \mathrm{b}$ & 2 & 1 & & 3 & 2 & & Tolerant \\
\hline$(\mathrm{Ma} / \mathrm{b})^{*}(\mathrm{Cr} / \mathrm{b}), \mathrm{F}_{4}$ & 2 & & 1 & 3 & & 2 & Tolerant \\
\hline $\mathrm{La} / \mathrm{b}$ & 2 & & 2 & 4 & & 3 & Tolerant \\
\hline $\mathrm{Ka} / \mathrm{b}$ & 2 & & 3 & 5 & & 4 & Moderate \\
\hline$(\mathrm{Ka} / \mathrm{b}) *(\mathrm{Cr} / \mathrm{b}), \mathrm{F}_{2}$ & 3 & & 2 & 5 & 4 & & Moderate \\
\hline$(\mathrm{Ka} / \mathrm{b})^{*}(\mathrm{Cr} / \mathrm{b}), \mathrm{F}_{6}$ & 3 & & 2 & 5 & 4 & & Moderate \\
\hline $\mathrm{Cr} / \mathrm{b}$ & 3 & & 3 & 6 & 5 & & Sensitive \\
\hline $\mathrm{La}(4 \mathrm{~b}, 4 \mathrm{~d}) / \mathrm{b}$ & 4 & & 4 & 8 & 5 & & Sensitive \\
\hline
\end{tabular}

Table 5. Rankings of genotypes for their relative salt tolerance based on grain yield per plant in green house experiment.

\begin{tabular}{|c|c|c|c|c|c|c|}
\hline \multirow[t]{2}{*}{ Genotypes } & \multicolumn{3}{|c|}{ Salinity levels (mMNaCl) } & \multirow[t]{2}{*}{ Sum } & \multirow{2}{*}{$\begin{array}{r}\text { Genotype } \\
\text { ranking }\end{array}$} & \multirow[t]{2}{*}{ Tolerance degree } \\
\hline & & 100 & 200 & & & \\
\hline$(\mathrm{St} / \mathrm{b})^{*}(\mathrm{Cr} / \mathrm{b}), \mathrm{F}_{4}$ & 1 & 2 & 3 & & 1 & Tolerant \\
\hline $\mathrm{Az} / \mathrm{b}$ & 2 & 1 & 3 & & 1 & Tolerant \\
\hline $\mathrm{Ka} / \mathrm{b}$ & 1 & 3 & 4 & & 2 & Moderate \\
\hline $\mathrm{La} / \mathrm{b}$ & 2 & 2 & 4 & & 2 & Moderate \\
\hline$(\mathrm{Ka} / \mathrm{b})^{*}(\mathrm{Cr} / \mathrm{b}), \mathrm{F}_{2}$ & 2 & 2 & 4 & & 2 & Moderate \\
\hline$(\mathrm{Ka} / \mathrm{b})^{*}(\mathrm{Cr} / \mathrm{b}), \mathrm{F}_{6}$ & 2 & 2 & 4 & & 2 & Moderate \\
\hline$(\mathrm{Ma} / \mathrm{b})^{*}(\mathrm{Cr} / \mathrm{b}), \mathrm{F}_{4}$ & 2 & 3 & 5 & & 3 & Moderate \\
\hline $\mathrm{Cr} / \mathrm{b}$ & 3 & 4 & 7 & & 4 & Sensitive \\
\hline $\mathrm{La}(4 \mathrm{~b}, 4 \mathrm{~d}) / \mathrm{b}$ & 4 & 5 & 9 & & 5 & Sensitive \\
\hline
\end{tabular}

Table 6. Effect of salinity treatment on reproductive traits of tritipyrum lines and wheat cultivar Bam in field conditions. Different letters indicate statistically different means ( $p \leq 0.05)$.

\begin{tabular}{|c|c|c|c|c|c|c|c|}
\hline Genotypes & $\begin{array}{r}\text { Salinity } \\
\text { level }\end{array}$ & $\begin{array}{l}\text { Number of } \\
\text { total tiller } \\
\text { per plant }\end{array}$ & $\begin{array}{l}\text { Number of } \\
\text { fertile tiller } \\
\text { per plant }\end{array}$ & $\begin{array}{l}\text { Number of } \\
\text { spike per } \\
\mathrm{m}^{2}\end{array}$ & $\begin{array}{l}\text { Spike } \\
\text { weight per } \\
\text { plant (g) }\end{array}$ & $\begin{array}{l}\text { Spike } \\
\text { length }(\mathrm{cm})\end{array}$ & $\begin{array}{lr}\begin{array}{l}\text { Number } \\
\text { spikelet }\end{array} & \text { per } \\
\text { spike } & \end{array}$ \\
\hline \multirow[t]{3}{*}{$\mathrm{Az} / \mathrm{b}$} & Control & $3.07^{\mathrm{de}}$ & $3.07^{\mathrm{ef}}$ & $223.07^{\mathrm{ef}}$ & $4.74^{\mathrm{cd}}$ & $12.69^{\mathrm{a}}$ & $12.93^{\mathrm{cd}}$ \\
\hline & $7 \mathrm{dS} / \mathrm{m}$ & $5.85^{\mathrm{ab}}$ & $5.60^{\mathrm{ac}}$ & $425.60^{\mathrm{ac}}$ & $9.16^{\mathrm{ab}}$ & $11.82^{\mathrm{bc}}$ & $12.07^{\mathrm{eg}}$ \\
\hline & $14 \mathrm{dS} / \mathrm{m}$ & $4.27^{\text {bd }}$ & $4.27^{\text {be }}$ & $324.27^{\text {be }}$ & $7.50^{\mathrm{ac}}$ & $11.35^{\mathrm{c}}$ & $11.53^{\mathrm{fg}}$ \\
\hline \multirow[t]{3}{*}{$(\mathrm{Ka} / \mathrm{b})^{*}(\mathrm{Cr} / \mathrm{b}), \mathrm{F}_{6}$} & Control & $4^{\text {cd }}$ & $4^{\mathrm{ce}}$ & $304.00^{\text {ce }}$ & $7.11^{\text {ac }}$ & $11.80^{b c}$ & $12.33^{\mathrm{df}}$ \\
\hline & $7 \mathrm{dS} / \mathrm{m}$ & $5.73^{\mathrm{ab}}$ & $5.73^{\mathrm{ab}}$ & $435.73^{\mathrm{ab}}$ & $9.38^{\mathrm{ab}}$ & $11.33^{c}$ & $13.07^{\mathrm{eg}}$ \\
\hline & $14 \mathrm{dS} / \mathrm{m}$ & $3.80^{\text {cd }}$ & $3.80^{\text {de }}$ & $288.80^{\text {de }}$ & $7.18^{\mathrm{ac}}$ & $10.49^{d}$ & $11.27^{\mathrm{g}}$ \\
\hline \multirow[t]{3}{*}{$(\mathrm{St} / \mathrm{b})^{*}(\mathrm{Cr} / \mathrm{b}), \mathrm{F} 4$} & Control & $5.33^{\mathrm{ac}}$ & $5.13^{\mathrm{bd}}$ & $390.13^{\text {bd }}$ & $7.70^{\mathrm{ac}}$ & $13.19^{\mathrm{a}}$ & $14.07^{\mathrm{ab}}$ \\
\hline & $7 \mathrm{dS} / \mathrm{m}$ & $6.80^{\mathrm{a}}$ & $6.80^{\mathrm{a}}$ & $516.80^{\mathrm{a}}$ & $9.96^{\mathrm{a}}$ & $12.91^{\mathrm{a}}$ & $13.53^{b c}$ \\
\hline & $14 \mathrm{dS} / \mathrm{m}$ & $3.93^{\text {cd }}$ & $3.93^{\mathrm{de}}$ & $298.93^{\text {de }}$ & $6.67^{\mathrm{ac}}$ & $12.55^{\mathrm{ab}}$ & $12.60^{\mathrm{de}}$ \\
\hline \multirow[t]{3}{*}{ Wheat cultivar Bam } & Control & $4.27^{\text {bd }}$ & $3.87^{\text {de }}$ & $293.87^{\text {de }}$ & $8.37^{\mathrm{ab}}$ & $10.27^{\text {de }}$ & $16.27^{\mathrm{a}}$ \\
\hline & $7 \mathrm{dS} / \mathrm{m}$ & $3.67^{\text {cd }}$ & $2.87^{\mathrm{ef}}$ & $217.87^{\mathrm{ef}}$ & $6.33^{\mathrm{bc}}$ & $9.62^{\mathrm{ef}}$ & $14.67^{\mathrm{a}}$ \\
\hline & $14 \mathrm{dS} / \mathrm{m}$ & $1.73^{\mathrm{e}}$ & $1.60^{f}$ & $121.60^{f}$ & $2.86^{d}$ & $9.40^{f}$ & $14.27^{\mathrm{ab}}$ \\
\hline \multirow[t]{2}{*}{$\mathrm{Az} / \mathrm{b}$} & Contro & $95.87^{b c}$ & $0.99^{\mathrm{ac}}$ & $0.27^{d}$ & $39.17^{\text {cd }}$ & $783.64^{\text {cd }}$ & $35.92^{\text {cd }}$ \\
\hline & $7 \mathrm{dS} / \mathrm{n}$ & $177.60^{\mathrm{a}}$ & $1.01^{\mathrm{a}}$ & $0.28^{\mathrm{cd}}$ & $43.07^{\mathrm{a}}$ & $1313.28^{\mathrm{ab}}$ & $44.59^{b c}$ \\
\hline
\end{tabular}




\begin{tabular}{|c|c|c|c|c|c|c|c|}
\hline & $14 \mathrm{dS} / \mathrm{m}$ & $139.87^{\mathrm{ab}}$ & $0.96^{\mathrm{ce}}$ & $0.27^{d}$ & $36.28^{\mathrm{ac}}$ & $1013.74^{\mathrm{bc}}$ & $38.12^{\mathrm{cd}}$ \\
\hline \multirow[t]{3}{*}{$(\mathrm{Ka} / \mathrm{b})^{*}(\mathrm{Cr} / \mathrm{b}), \mathrm{F}_{6}$} & Control & $134.53^{\mathrm{ab}}$ & $0.97^{\text {be }}$ & $0.26^{d}$ & $40.17^{\mathrm{ac}}$ & $1019.14^{b c}$ & $40.22^{\mathrm{bd}}$ \\
\hline & $7 \mathrm{dS} / \mathrm{m}$ & $180.13^{\mathrm{a}}$ & $0.99^{\mathrm{ab}}$ & $0.27^{d}$ & $41.29^{\mathrm{ab}}$ & $1314.68^{\mathrm{ab}}$ & $43.11^{\mathrm{dd}}$ \\
\hline & $14 \mathrm{dS} / \mathrm{m}$ & $132.07^{\mathrm{ab}}$ & $0.95^{\mathrm{de}}$ & $0.25^{\mathrm{d}}$ & $39.90^{\mathrm{ac}}$ & $1019.29^{b c}$ & $39.19^{\mathrm{bd}}$ \\
\hline \multirow[t]{3}{*}{$(\mathrm{St} / \mathrm{b})^{*}(\mathrm{Cr} / \mathrm{b}), \mathrm{F} 4$} & Control & $141.80^{\mathrm{ab}}$ & $0.95^{\mathrm{de}}$ & $0.25^{d}$ & $37.50^{\mathrm{ac}}$ & $1113.27^{\mathrm{ac}}$ & $36.27^{d}$ \\
\hline & $7 \mathrm{dS} / \mathrm{m}$ & $185.40^{\mathrm{a}}$ & $0.98^{\mathrm{ad}}$ & $0.26^{d}$ & $40.11^{\mathrm{ab}}$ & $1512.19^{\mathrm{a}}$ & $37.36^{\mathrm{cd}}$ \\
\hline & $14 \mathrm{dS} / \mathrm{m}$ & $129.20^{\mathrm{ab}}$ & $0.93^{\mathrm{e}}$ & $0.25^{d}$ & $36.32^{\mathrm{bd}}$ & $1049.24^{b c}$ & $33.91^{d}$ \\
\hline \multirow[t]{3}{*}{ Wheat cultivar Bam } & Control & $130.27^{\mathrm{ab}}$ & $0.75^{\dagger}$ & $0.40^{\mathrm{a}}$ & $53.07^{\mathrm{ab}}$ & $916.45^{b c}$ & $57.20^{\mathrm{a}}$ \\
\hline & $7 \mathrm{dS} / \mathrm{m}$ & $104.57^{b c}$ & $0.74^{\dagger}$ & $0.34^{\mathrm{b}}$ & $45.12^{\mathrm{bc}}$ & $699.52^{\mathrm{cd}}$ & $51.22^{\mathrm{ab}}$ \\
\hline & $14 \mathrm{dS} / \mathrm{m}$ & $43.37^{c}$ & $0.72^{f}$ & $0.33^{\mathrm{bc}}$ & $42.98^{\mathrm{d}}$ & $310.38^{d}$ & $45.51^{\text {bd }}$ \\
\hline
\end{tabular}

\begin{tabular}{|c|c|c|c|}
\hline $\begin{array}{l}\text { Abbreviation, ploidy level and genomic constitution of tritipyrum lines } \\
\text { and wheat cultivar in the present study.Genotypes }\end{array}$ & Abbreviation & Ploidy & Genome \\
\hline \multicolumn{4}{|l|}{ Wheat cultivars } \\
\hline T.aestivum cv. Bam & Bam & $6 \mathrm{X}$ & AABBDD \\
\hline \multicolumn{4}{|l|}{ Tritipyrum lines } \\
\hline$($ Karim/Th. Bessarabicum $) \times($ Creso/ Th. Bessarabicum $) \mathrm{F}_{6}$ & $(\mathrm{Ka} / \mathrm{b})^{*}(\mathrm{Cr} / \mathrm{b}), \mathrm{F}_{6}$ & $6 \mathrm{X}$ & $A A B B E^{b} E^{b}$ \\
\hline (Karim/Th. Bessarabicum $) \times\left(\right.$ Creso/ Th. Bessarabicum) $\mathrm{F}_{2}$ & $(\mathrm{Ka} / \mathrm{b})^{*}(\mathrm{Cr} / \mathrm{b}), \mathrm{F}_{2}$ & $6 \mathrm{X}$ & $A A B B E^{b} E^{b}$ \\
\hline (Macoun/ Th. Bessarabicum $) \times\left(\right.$ Creso/Th. Bessarabicum) $\mathrm{F}_{4}$ & $(\mathrm{Ma} / \mathrm{b})^{*}(\mathrm{Cr} / \mathrm{b}), \mathrm{F}_{4}$ & $6 \mathrm{X}$ & $A A B B E^{b} E^{b}$ \\
\hline (Stewart/ Th. Bessarabicum) $\times\left(\right.$ Creso/ Th. Bessarabicum) $\mathrm{F}_{4}$ & $(\mathrm{St} / \mathrm{b})^{*}(\mathrm{Cr} / \mathrm{b}), \mathrm{F}_{4}$ & $6 \mathrm{X}$ & $A A B B E^{b} E^{b}$ \\
\hline Langdon/ Th. Bessarabicum (4B,4D)/ Th. Bessarabicum & $\mathrm{La}(4 \mathrm{~B}, 4 \mathrm{D}) / \mathrm{b}$ & $6 X$ & $A A B B E^{b} E^{b}$ \\
\hline Karim/Th. Bessarabicum & $\mathrm{Ka} / \mathrm{b}$ & $6 x$ & $A A B B E^{b} E^{b}$ \\
\hline Creso/ Th. Bessarabicum & $\mathrm{Cr} / \mathrm{b}$ & $6 \mathrm{X}$ & $A A B B E^{b} E^{b}$ \\
\hline Langdon/ Th. Bessarabicum & $\mathrm{La} / \mathrm{b}$ & $6 x$ & $A A B B E^{b} E^{b}$ \\
\hline Aziziah/Th. Bessarabicum & $\mathrm{Az} / \mathrm{b}$ & $6 \mathrm{X}$ & $A A B B E^{b} E^{b}$ \\
\hline
\end{tabular}

as to produce higher grain yield before and after anthesis (El-Hendawy et al., 2009; Zeng et al., 2003). In the hydrocultural experiment conducted in the current study, flag leaf area and length decreased following an increase in salinity (Table 1). Additionally, salinity stress decreased the number of leaf per main stem in tritipyrum lines (Table 1). Although salinity caused some changes in the characteristics of leaves, there was no significant difference between the number of leaves per main stem for salt-tolerant and saltsensitive tritipyrum lines (Table 1). Thus, it may be concluded that the characteristics of leaves in tritipyrum lines (e.g. flag leaf length and area) could be used as reliable indicators for salt tolerance of tritipyrum lines under salinity stress. The findings of this study with regards to leaves' characteristics were in line with El-Hendawy et al., (2009) who introduced leaves traits as a reliable criterion for determining the degree of salt tolerance.

According to the results of this study, growth and development of leaves and their area decreased due to salinity. In fact, this may be a reason for the reduction of grain yield during salinity stress through photo assimilate and biomass reduction (Afifi et al., 2010). The height of the main stems of tritipyrum lines decreased as a result of salinity stress. The sensitive lines were shorter than the tolerant ones (Table 1). During salinity stress, stem length showed reduction by about $17.5 \%$ and $10 \%$ in sensitive and tolerant lines, respectively. Decreased plant height following salinity stress has also been reported by other researchers (e.g. Hasanuzzaman et al., 2009). The inhibitory effect of salinity on cell division and elongation have been mentioned as reasons for the decrease in stem length during plant development under salinity stress (Munns and Tester, 2008). Furthermore, the number of tillers in sensitive tritipyrum lines showed greater reduction (about $43.5 \%$ ) compared to salt-tolerant lines (about 26.3\%). Thus, these traits can be used as reliable criteria to determine salt tolerance among tritipyrum lines. Since the number of tillers determines the number of bearing spike, it was used as to estimate grain yield of wheat genotype. It was shown that salinity decreases tiller production by preventing their formation or leading to their death (Nicolas et al., 1993). Therefore, the number of tillers and their behavior during salinity stress were identified as a salt-tolerant mechanism and valuable selection criteria for screening wheat salt tolerance in breeding programs (Zeng and Shannon, 2000; El-Hendawy et al., 2009; Zeng et al., 2003). Cluster analysis based on the above phenotypic traits and multivariate parameters was identified as a convenient and accurate method for evaluating salt tolerance and ranking wheat and rice genotypes (Zeng et al., 2002; El-Hendawy et al., 2005). Accordingly, this method was used for estimating salt tolerance of tritipyrum lines in this experiment and leaf, stem and tiller characteristics were used to determine the salt tolerance of tritipyrum lines at the vegetative growth stage. Based on these traits, $(\mathrm{St} / \mathrm{b}) \times(\mathrm{Cr} / \mathrm{b}), \mathrm{F} 4, \mathrm{Az} / \mathrm{b}$, $(\mathrm{Ma} / \mathrm{b}) \times(\mathrm{Cr} / \mathrm{b}), \mathrm{F} 4$ and $\mathrm{La} / \mathrm{b}$ were found to be salt-tolerant lines (Table 3).

Among the measured yield components and reproductive traits, the number of spikelet per spike and spike length decreased following an increase in salinity. On the contrary, the number of grains and single grain weight increased proportionate to any increase in salinity up to $100 \mathrm{mM} \mathrm{NaCl}$ and the amount of these traits decreased after increasing salinity up to $200 \mathrm{mM} \mathrm{NaCl}$ (Table 1). Cluster analysis based on these traits introduced $(\mathrm{St} / \mathrm{b}) \times(\mathrm{Cr} / \mathrm{b}), \mathrm{F} 4, \mathrm{Az} / \mathrm{b}$, $(\mathrm{Ma} / \mathrm{b}) \times(\mathrm{Cr} / \mathrm{b}), \mathrm{F} 4$ and $\mathrm{La} / \mathrm{b}$ as salt-tolerant tritipyrum lines (Table 4). According to the obtained results, there was no significant difference between salt-tolerant and salt- 
sensitive tritipyrum lines with regards to the percentage of reduction in the number of spikelet per spike, spike length and number of grains per plant. These results are consistent with the findings of El- Hendavy et al., (2005) and Zeng et al., (2002) who have reported that the number of grain per plant is a poor selecting criterion for determining the salttolerance of rice and wheat under control condition. However, other researchers have found that reduction in the number of grains causes reduction in the grain yield of wheat and they have accordingly introduced it as a suitable screening criterion for the salt tolerance of wheat (Ahmad et al., 2005; Grewal, 2010). Moreover, the number of spikelet per spike has shown to be an effective criterion for screening wheat genotypes in terms of salt tolerance and this trait had indeed a highly significant correlation with grain yield (ElHendawy et al., 2009). In contrast with our finding, Hasanuzzaman et al., (2009) reported that in rice, panicle length was affected by salinity stress. Reduction of single seed weight was $10.12 \%$ and $39.31 \%$ in sensitive and tolerant tritipyrum lines, respectively. The increase in some of the measured traits due to increased salinity may point to the fact that tritipyrum is potentially a halophyte cereal (Table 1). It has also been reported that grain weight has a significant effect on grain yield and that a strong correlation was observed between grain weight and grain yield in rice (Ahmad et al., 2005).

Grain yield of wheat is a determining criterion for salt tolerance. Therefore, it is an important target in almost all breeding programs (Ashraf, 2004; Reynolds et al., 2010). Grain yield is affected not only by yield components such as the number of spikes, number of spikelet per spike and grain weight, but also by agronomic traits including leaves' characteristics and number of tillers. These traits are affected by salinity stress and they can hence be used to determine the salt tolerance of wheat at different growth stages (Saqib et al., 2004). Cluster analysis based on grain yield distinguished $(\mathrm{St} / \mathrm{b}) \times(\mathrm{Cr} / \mathrm{b}), \mathrm{F} 4$ and $\mathrm{Az} / \mathrm{b}$ as salt-tolerant lines (Table 5). Grain yield of salt-tolerant tritipyrum lines increased with an increase in salinity up to $100 \mathrm{mM}$, while grain yield of salt-sensitive lines experienced a decreasing trend (Fig. 1). At $200 \mathrm{mM} \mathrm{NaCl}$, the grain yields of salttolerant and salt-sensitive lines decreased by $20.3 \%$ and $55.3 \%$ compared to $50 \mathrm{mM} \mathrm{NaCl}$, respectively.

Generally, in greenhouse experiments, $(\mathrm{St} / \mathrm{b}) \times(\mathrm{Cr} / \mathrm{b}), \mathrm{F} 4$ and $A z / b$ are known to be salt-tolerant at all growth stages. In the current study, these genotypes had the highest amount of flag leaf length, flag leaf area, leaf number per main stem, main stem height and tiller number per plant at the vegetative stage (Table 1 ). In addition, they had the highest spikelet per spike, spike length, seed number per plant and single seed weight at the reproductive stage (Table 1). Other lines were also ranked as salt-tolerant at different growth stages, but the grain yield of $(\mathrm{St} / \mathrm{b}) \times(\mathrm{Cr} / \mathrm{b}), \mathrm{F} 4$ and $\mathrm{Az} / \mathrm{b}$ was higher than the other lines under salinity stress condition (Fig. 1). Thus, according to the results of the hydrocultural experiment, $(\mathrm{St} / \mathrm{b}) \times(\mathrm{Cr} / \mathrm{b}), \mathrm{F} 4$ and $\mathrm{Az} / \mathrm{b}$ can be included in plant breeding programs in order to improve the salt tolerance of wheat genotypes.

Growth conditions in the field are more complicated than the greenhouse condition and these complexities can potentially affect traits such as yield and it may ultimately limit the divination of the field performance of tritipyrum. Agronomic traits (Table 6 and Fig. 2-3), grain yield (Fig. 3B) and the amount of grain protein of tritipyrum lines (Fig. 3C) were measured and the data indicated that tritipyrum lines are superior to Bam wheat cultivar in saline soil in the field condition. Therefore, the field results supplied strong evidence regarding the positive contribution of these lines for improving agricultural productivity in saline conditions.

\section{Materials and methods}

The greenhouse experiment and filed trail were both conducted at the Faculty of Agriculture, Ferdowsi University of Mashhad, Iran, during 2013/2014 and 2014/2015, respectively.

\section{Greenhouse experiment}

Genetic materials used in this study included nine hexaploid tritipyrum lines derived from durum wheat cultivar and Thinopyrum Bessarabicum by King et al., (1997). Tritipyrum lines including $(\mathrm{Ka} / \mathrm{b}) \times(\mathrm{Cr} / \mathrm{b}), \quad \mathrm{F} 6,(\mathrm{Ka} / \mathrm{b}) \times(\mathrm{Cr} / \mathrm{b}), \quad \mathrm{F} 2$, $(\mathrm{Ma} / \mathrm{b}) \times(\mathrm{Cr} / \mathrm{b}), \mathrm{F} 4,(\mathrm{St} / \mathrm{b}) \times(\mathrm{Cr} / \mathrm{b}), \mathrm{F} 4, \mathrm{La}(4 \mathrm{~B}, 4 \mathrm{D}) / \mathrm{b}, \mathrm{Cr} / \mathrm{b}, \mathrm{Az} / \mathrm{b}$, $\mathrm{La} / \mathrm{b}$, and $\mathrm{Ka} / \mathrm{b}$ were used in this experiment (Table 1 ). Furthermore, Iranian salt-tolerant Bam wheat cultivar was used as the standard (Table 7).

In order to vernalize tritipyrum lines, uniform seeds in terms of shape and weight were selected and sown in seedling growing trays outside the greenhouse for six weeks. Then, healthy and similar seedlings in terms of shape and size were selected and cultivated in planting containers $(43 \times 25 \times 34 \mathrm{~cm})$ (Supplementary Fig. 2) and then transferred to a greenhouse with a daily temperature of $25^{\circ} \mathrm{C} / 20^{\circ} \mathrm{C}$ $\mathrm{day} / \mathrm{night}$ and natural light without relative humidity control in a sand culture system. In each container, 4 rows of seedlings were planted with $8 \mathrm{~cm}$ spacing between the rows and $2 \mathrm{~cm}$ spacing between the seedlings. Irrigation was arranged through three irrigation pipes that were connected to a main water pump. The pumps were inserted in 100 liters plastic barrels with half strength Hoagland nutrient solution (Hoagland \&Arnon 1950). After three days, the seedlings received full strength Hoagland solution. Salt treatments were applied incrementally at the 4th leaf stage until maturity level (Supplementary Fig. 2). To perform the salt treatments, $\mathrm{NaCl}$ was added in 3 steps by $50 \mathrm{mM}$ $\mathrm{NaCl} /$ day to reach the intended salinity levels, i.e. 50, 100 and $200 \mathrm{mMNaCl}$. Control plants received $50 \mathrm{mMNaCl}$ that is very similar to the salinity level of irrigation water in many parts of Iran. All the plants were watered daily by pumping Hoagland's solution containing 50,100 and $200 \mathrm{mMNaCl}$ and the solutions were renewed on a weekly basis. The genotypes were grown until reaching maturity level (Supplementary Fig. 2).

At $50 \%$ of complete spikes emergence stage, five plants of each genotype were harvested and their vegetative traits including flag leaf length and area, number of leaves per main stem, main stem length and number of tillers were measured. At the maturity stage, the spikes of the main stems of five treated plants were harvested and their length, number of spikelet per spike, length and width of the grains, single seed weight and number of seeds per plant were measured. The grain yield of genotypes was calculated by weighting the grains of all plant tillers.

Following Zeng et al., (2002) and El- Henawy et al., (2005), all the data were converted into salt tolerance indices 
defined as the observation at salinity divided by the average of the controls. Then, cluster analysis of the salt tolerance indices was arranged based on Ward's method. The cluster analysis ranked genotypes at the vegetative stage based on the number of tillers, number of leaves per main stem, flag leaf length and area and main stem length. Spike length, number of spikelet per spike, length and width of grains, single seed weight, number of grains per plant and seed weight per plant were used for cluster analysis to rank the genotypes for salinity stress at the reproductive stage. Additionally, the genotypes were clustered based on grain yield per plant. The number of cluster groups at each salinity level in each genotype was summarized and the genotypes were ranked based on the sums.

\section{Field experiment}

Field experiment for salt tolerance was performed at the research field of the Faculty of Agriculture of Ferdowsi University of Mashhad, Khorasan Razavi province located in East Iran with a latitude of $36^{\circ} 18^{\prime} \mathrm{N}$, longitude of $59^{\circ} 35^{\prime} \mathrm{E}$ and $1050 \mathrm{~m}$ altitude above sea level (Supplementary Fig. 3).

Based on the results of the greenhouse experiment, two salt-tolerant tritipyrum lines $((\mathrm{St} / \mathrm{b}) \times(\mathrm{Cr} / \mathrm{b}), \mathrm{F} 4, \mathrm{Az} / \mathrm{b})$, one semi-salt tolerant tritipyrum line $((\mathrm{Ka} / \mathrm{b}) \times(\mathrm{Cr} / \mathrm{b}), \mathrm{F} 4)$ and $\mathrm{a}$ salt-tolerant wheat cultivar (Bam) were selected for field experiment. The main goal of the greenhouse experiment was to address the question of whether tritipyrum lines are more salt-tolerant than wheat. Therefore, to avoid waste of time and reduce labor and costs, control treatment was not considered and salinity levels were merely applied in the greenhouse experiment. Assured that tritipyrum lines were more salt-tolerant than wheat, control treatment (without salinity) was considered in the field experiment similar to other researches. Hoagland's solution increases salinity by about 1- $2 \mathrm{dS} / \mathrm{m}$, Furthermore, water salinity in most regions of Iran is about $7 \mathrm{dS} / \mathrm{m}$; hence, salinity treatments in the field experiment were similarly considered for the control treatment, 7 and $14 \mathrm{dS} / \mathrm{m}$. The genotypes were irrigated weekly by farm water containing $0.98 \mathrm{dS} / \mathrm{m}$ of EC until stem elongation. Then, irrigation was done by water containing $0.98 \mathrm{dS} / \mathrm{m}$ of EC as the control and water containing $7 \mathrm{dS} / \mathrm{m}$ and $14 \mathrm{dS} / \mathrm{m}$ of EC as salinity treatments. The size of the plots was $2 m \times 4 m$, consisted of four rows from each genotype in 2-m length. The space between the rows was 50 $\mathrm{cm}$ and the gap between the plots was $1 \mathrm{~m}$. In order to record the agronomical and morphological traits at the maturity stage, ten plants were randomly sampled from each genotype in plots and plant height (the height from the ground to the tip of the main spike in $\mathrm{cm}$ ), peduncle length (average height of peduncle which is the last inter-node of the main stem in $\mathrm{cm}$ ), spike length (from the base of the spike to the end of the spike in $\mathrm{cm}$ ), spike weight per plant, number of spikelet per spike, number of seeds per plant, width and length of grains, total number of tillers per plant, number of fertile tiller per plant and straw weight per plant were measured. Furthermore, number of spikes per square meter, 1000 grains' weight, biological yield (the total amount of shoot dry biomass of plants per square meter), harvest index (grain yield/ biological yield* 100), and grain yield per square meter were measured in each plot of the treatment. Also, grain protein content was calculated through Kjeldahl's method (\%N×回5.7).

\section{Experimental design and statistical analysis}

The experiment was arranged in a split plot based on a completely randomized design and split plot based on randomized block design with salinity as the main plots and genotypes as sub-plots with three replicates, in greenhouse and field conditions, respectively. Simple statistical analysis including mean comparison and standard error were conducted by SPSS (Ver. 16.0, SPSS, Chicago, IL, USA). Mean comparisons were calculated using Tukey's test at $5 \%$ probability level. Cluster analysis was carried out using Statgraphics Centurion software (version 16) and the charts were drawn using Microsoft Excel.

\section{Conclusion}

Overall, the results obtained in this study clearly pointed to the superiority of the qualitative and quantitative traits of tritipyrum lines compared to wheat in saline conditions. These results are promising in that they show the potential for improving the salinity tolerance of wheat through breeding programs. Also, tritipyrum lines can be potentially introduced as a long-term solution for producing cereal in highly saline soils or saline irrigation water worldwide. However, it has to be noticed that tritipyrum is a new salttolerant cereal and still more breeding efforts are required to produce commercial tritipyrum cultivar.

\section{Acknowledgments}

The authors would like to express their appreciation to Ferdowsi University of Mashhad for funding this research under grant No. 29653. We would also like to extend our sincere gratitude to our colleague, Dr. Shahsavand Hassani, for providing us with the seeds of tritiprym lines.

\section{References}

Afifi M, Saker M, Ahmed M, Khatab S (2010) Morphological and Physiological Studies on the effect of salinity and growth promoters on rice plants. Acta Agron Hung. 58: 1120.

Ahmad M, Niazi B, Zaman B, Athar M (2005) Varietals differences in agronomic performance of six wheat varieties grown under saline field environment. Int $\mathrm{J}$ Environ Sci Te. 2: 49-57.

Ashraf M (2004) Some important physiological selection criteria for salt tolerance in plants. Flora. 199: 361-376.

Ashraf M, Athar H, Harris P, Kwon T (2008) Some prospective strategies for improving crop salt tolerance. Adv Agron. 97: 45-110.

Colmer TD, Flowers TJ, Munns R (2006) Use of wild relatives to improve salt tolerance in wheat. J Exp Bot. 57: 10591078.

El-Hendawy SE, Hu Y, Yakout GM, Awad AM, Hafiz SE, Schmidhalter $U$ (2005) Evaluating salt tolerance of wheat genotypes using multiple parameters. Eur J Agron. 22: 243-253.

El-Hendawy SE, Ruan Y, Hu Y, Schmidhalter U (2009) A comparison of screening criteria for salt tolerance in wheat under field and controlled environmental conditions. J Agron Crop Sci. 195: 356-367.

FAO.FAO Agristat. http://www.fao.org/, 2007 
Grewal HS (2010) Response of wheat to subsoil salinity and temporary water stress at different stages of the reproductive phase. Plant Soil. 330: 103-113.

Hasanuzzaman M, Fujita M, Islam M, Ahamed K, Nahar K (2009) Performance of four irrigated rice varieties under different levels of salinity stress. Int J Integr Biol. 16: 85-90.

Hoagland DR, Arnon DI (1950) The water-culture method for growing plants without soil, Circular. Calif Aes Bull. 347: 23-32.

Kamyab M, Kafi M, Shahsavand H, Goldani M, Shokouhifar F (2016) Exploring ion homeostasis and mechanism of salinity tolerance in primary tritipyrum lines (wheat thinopyrum bessarabicum) in the presence of salinity. Aust J Crop Sci. 10: 911-919.

King I, Law C, Cant K, Orford S, Reader S, Miller T (1997) Tritipyrum, a potential new salt-tolerant cereal. Plant Breeding. 116: 127-132.

Läuchli A, Grattan SR (2007) Plant growth and development under salinity stress. In: Jenks MA, Hasegawa PA, Jain SM (eds) Advances in molecular breeding toward salinity and drought tolerance. Springer-Verlag, NewYork. 1-32.

Munns R, Tester M (2008) Mechanisms of salinity tolerance. Annu Rev Plant Biol. 59: 651-681.

Nicolas M, Munns R, Samarakoon A, Gifford R (1993) Elevated $\mathrm{CO} 2$ improves the growth of wheat under salinity. Funct Plant Biol. 20: 349-360.
Parida AK, Das AB (2005) Salt tolerance and salinity effects on plants: a review. Ecotox Environ Safe. 60: 324-349.

Qadir M, Oster J (2004) Crop and irrigation management strategies for saline-sodic soilsand waters aimed at environmentally sustainable agriculture. Sci Total Environ. 323: 1-19.

Reynolds M, Bonnett D, Chapman SC, Furbank RT, Manès $Y$, Mather DE, Parry MA (2010) Raising yield potential of wheat. I. Overview of a consortium approach and breeding strategies. J Exp Bot. 62: 439-452.

Saqib M, Akhtar J, Qureshi RH (2004) Pot study on wheat growth in saline and waterlogged compacted soil: I. Grain yield and yield components. Soil Till Res. 77: 169-177.

Singh S, Singh M (2000) Genotypic basis of response to saliniity stress in some crosses of spring wheat Triticum aestivum L. Euphytica. 115: 209-214.

Zeng L, Poss JA, Wilson C, Draz ASE, Gregorio GB, Grieve CM (2003) Evaluation of salt tolerance in rice genotypes by physiological characters. Euphytica. 129: 281-292.

Zeng L, Shannon MC (2000) Salinity effects on seedling growth and yield components of rice. Crop Sci. 40: 9961003.

Zeng L, Shannon M, Grieve C (2002) Evaluation of salt tolerance in rice genotypes by multiple agronomic parameters. Euphytica. 127:235-245. 